\title{
MODELO EDUCATIVO \\ PARA EL PERSONAL DE ENFERMERÍA \\ Diseño y aplicación
}

Enfermera Rocio Moreno de Santa Cruz *

Coautoras: Xiomara Alicia Alvarez Pérez, Ligia Fernanda Ariza Lopez, Lorena Judith Daza Campos, Diana Yineth Jimenez

Castillo, Nohora Milena Rincón Pardo, Andrea Carolina Sepúlveda González, Alejandra Vásquez **

\section{Resumen}

Este estudio se realizó con el objetivo de estandarizar la práctica educativa del personal de enfermería del pabellón Fundadores A del Hospital de San José de Bogotá con el paciente, acerca del cuidado en casa con la herida quirúrgica a través del diseño e implementación de un modelo educativo.

El diseño metodológico utilizado fue investigación-acción, el cual permite la interrelación de apoyo y asesoría con la población del estudio, así como la generación de nuevos conocimientos al investigador y a los grupos participantes.

Este fue un proceso dirigido por etapas. Primero se sensibilizó al personal de enfermería del pabellón Fundadores A, donde conocieron la importancia y la necesidad de implementar el modelo educativo. Con la participación del personal del estudio y del grupo investigador se diseñó el modelo, para seguir con el segundo paso del proceso que abrió el camino a la aplicación y evaluación del mismo.

La aplicación del diseño fue considerada por el personal como una herramienta de trabajo que unifica conocimientos y actividades designadas. El resultado evidencia que el grupo no solo recibió los conocimientos del modelo sino que lo convirtieron en parte de su práctica diaria.

En conclusión, el modelo educativo mejoró la práctica educativa del personal de enfermería con el paciente de cirugía, sobre los cuidados en casa con la herida quirúrgica. Esto se incluirá en el esquema de calidad que destaca a la institución.

Palabras claves: diseño, implementar, evaluar, modelo, estandarizar, práctica educativa, cuidado.

\section{Introducción}

En el caminar de la historia el cuidado de enfermería ha traspasado el concepto de ayuda a los demás para dar origen a los cuidados profesionales, dominio central de esta disciplina. Teniendo en cuenta que el Hospital de San Jose es una entidad docente asistencial donde las estrategias para mejorar la atención y disminuir los costos se basan en la calidad del servicio, nace la necesidad de implementar métodos educativos que involucren al paciente, la familia y al personal de salud en forma activa, buscando como

\footnotetext{
Enfermera jefe de Gestión, Comité de Infecciones, Hospital de San José, Bogotá.

* Comité de infecciones y personal del pabellón Fundadores A del Hospital de San José.
}

resultado la satisfacción del enfermo con el servicio recibido, la disminución del riesgo de complicaciones y la buena imagen institucional.

Siendo responsabilidad del personal de enfermería el cuidado integral del paciente y por tanto su educación, se ve la necesidad y la importancia de adoptar mecanismos satisfactorios para optimizar la calidad de la práctica educativa, teniendo en cuenta que la educación ejerce un papel importante en el mejoramiento de la calidad de vida y de la cultura.

El presente trabajo describe, a través del método acción participación, el diseño, aplicación y evaluación de un modelo educativo, cuyo objetivo es mejorar la práctica educativa en el personal de enfermería. 


\section{Métodos}

\section{Diseño metodológico}

Tipo de estudio: la investigación se llevó a cabo por medio de un estudio de investigación-acción que permite ligar la investigación cuantitativa con la cualitativa, resaltando su visión práctico-deliberativa, donde la interrelación de apoyo y asesoría con el personal de enfermería, permite la generación de nuevos conocimientos al investigador y a los grupos involucrados.

Población de estudio: el total fue de 80 enfermeras profesionales y auxiliares del HSJ, que laboraron durante el periodo de julio a octubre del 2004 en las áreas de manejo de pacientes quirúrgicos.

Muestra: Fueron cinco enfermeras y nueve auxiliares que laboraron en el pabellón Fundadores Adel HSJ en el mismo período. Fueron seleccionadas por medio de un muestreo no probabilístico a conveniencia.

Métodos e instrumentos de medición para la evaluación del modelo para la estandarización de la práctica educativa: teniendo en cuenta que una mejor práctica educativa implica un cambio cultural en el hacer y en el sentir del personal de enfermería, el grupo investigador definió tres instrumentos para la recolección de datos y análisis de los resultados de la investigación.

1) Escala de Likert: mide el grado de actitud que el personal de enfermería participante manifiesta frente a la estandarización de la práctica educativa a través del diseño y aplicación de un modelo. Esta conformada por 15 preguntas con tres opciones de respuesta cada una, categorizadas de la siguiente forma: a) de acuerdo, b) ni de acuerdo ni en desacuerdo y c) en desacuerdo.

2) Test de conocimientos sobre el diseño del modelo educativo: evalúa el aprendizaje de cada uno de los participantes sobre el diseño definido del modelo educativo. Consta de diez preguntas de selección múltiple con única respuesta y tres distractores, basados en qué, quién, cómo y cuándo se debe realizar la práctica educativa.
3) Lista de chequeo de la práctica educativa: describe el grado de modificación de la práctica educativa después de la aplicación del modelo por el personal de enfermería del pabellón Fundadores "A" del HSJ. Está conformada por diez preguntas: 1 y 2 con cuatro opciones de respuesta ( 1,1 a 1,4 y 2,1 a 2,4 respectivamente), la 3 con seis opciones $(3,1$ a 3,6$)$ y de la pregunta 4 a la 10 con dos (si, no).

Análisis estadístico: promedio, mediana y desviación estándar.

Criterios de inclusión: a) todo el personal de enfermería que haya participado y recibido el entrenamiento para el diseño y la aplicación del modelo educativo; b) personal que labore en el sitio asignado para la ejecución de la investigación pabellón Fundadores A y que tenga bajo su cuidado pacientes con herida quirúrgica.

Criterios de exclusión: a) Personal de enfermería que sea retirado del pabellón Fundadores. b) Personal de enfermería que se encuentre vinculado al comité de infecciones o clínica de heridas. c) Personal de enfermería a quien no fue posible la aplicación de los tres instrumentos de verificación: pruebas individuales de conocimientos y actitud hacia el modelo educativo y una colectiva sobre la lista de comprobación de la práctica educativa con el paciente quirúrgico sobre los cuidados en casa con la herida quirúrgica.

\section{Resultados}

Luego de la aplicación del modelo educativo y la evaluación del mismo, de acuerdo con lo estipulado en el cuadro de medición de variables, en la prueba de conocimientos se observa que el personal de enfermería evaluado se ubicó en un rango de bueno con una calificación promedio de 4,7 en una escala de 1 a 5 y la mediana de 5,0. Esto indica que por lo menos el $50 \%$ de la muestra seleccionada se encuentra con un puntaje igual a este. La desviación estándar refleja la homogeneidad de conocimientos en cuanto al modelo educativo implementado por el 
grupo participante (Tabla 1). Por lo anterior, los mayores avances de esta investigación se lograron en la variable de conocimientos del modelo educativo.

El personal tiene una actitud POSITIVA frente al modelo que se evidenció en la prueba de actitud con un promedio de 4.0 en una escala de 1 a 5 según lo planteado en el cuadro de medición de variables. A pesar de que en la desviación estándar el resultado no es significativo $(0,56)$, demuestra que en la mayoría del personal de enfermeria hay satisfacción con el modelo planteado. La pregunta 15 revela que el personal requiere supervisión permanente en la aplicación que garantice la continuidad. Esto incidió en la dispersión de resultados.

Como en el estudio anterior se aplicó una lista de comprobación donde solo se aplicaron los ítems que evaluaban la práctica educativa del personal de enfermería, este resultado evidencia que el grupo modificó la práctica cumpliendo con las actividades propuestas por el modelo. El promedio fue 4,3 en una escala de cumplimiento de 1 a 5 . La desviación estándar con un puntaje de 0,5 refleja que hay aspectos que deben ser reforzados en el grupo participante como la inclusión de la familia en la educación, valorado en el ítem 8 y la verificación del proceso educativo al paciente valorado en el ítem 7 de la lista de comprobación.

\section{Discusión}

Al finalizar el estudio se concluyó que la actitud es un factor central en la práctica educativa que evidencia los conocimientos contenidos en el recurso humano del pabellón Fundadores A. Luego de la aplicación del modelo educativo y la evaluación del mismo, en la prueba de conocimientos se observa que el personal de enfermería se ubicó en un rango bueno, lo cual señala que por lo menos el $50 \%$ de la muestra seleccionada se encuentra con un puntaje igual a este. La desviación estándar refleja la homogeneidad de conocimientos en cuanto al modelo educativo implementado por el grupo participante.

En cuanto a la prueba de actitud, se encontró un promedio de 4.0 en una escala de 1 a 5 , por lo que se puede afirmar que el personal de enfermería tiene una actitud POSITIVA frente al modelo educativo, resultado del estimulo y vigilancia que hizo posible ejercer control sobre la conducta basados en la teoría de B. F. Skinner 3 cuando afirma que el ser humano responde y aprende por los refuerzos.

Por último se aplicó la lista de comprobación validada y utilizada también en el estudio anterior de CAP (Conocimientos Actitudes y Prácticas) dando como resultado un promedio de 4,3 en una escala de cumplimiento de 1 a 5 , lo que permite afirmar que el personal de enfermería modificó la práctica educativa, cumpliendo la mayoría de las actividades propuestas por el modelo educativo.

Como en la exposición del modelo desarrollado por Knowles (1970) para el aprendizaje de los adultos, estos resultados determinan la capacidad de

\begin{tabular}{|l|c|c|c|c|c|}
\hline \multicolumn{6}{|c|}{$\begin{array}{c}\text { Tabla I. Estadistica descriptiva del diseño y aplicación del modelo educativo para la práctica educativa } \\
\text { del personal de enfermería del HSJ sobre los cuidados en casa con la herida quirúrgica }\end{array}$} \\
\hline Instrumento & $\begin{array}{c}\text { No. de } \\
\text { casos }\end{array}$ & Promedio & Porcentaje & Mediana & $\begin{array}{c}\text { Desviación } \\
\text { estándar }\end{array}$ \\
\hline $\begin{array}{l}\text { Prueba de } \\
\text { conocimientos }\end{array}$ & 14 & 4,7 & $94 \%$ & 5,0 & 0,37 \\
\hline Prueba de actitud & 14 & 4,0 & $80 \%$ & 4,0 & 0,56 \\
\hline $\begin{array}{l}\text { Lista de } \\
\text { comprobación }\end{array}$ & 5 & 4,3 & $86 \%$ & 4,5 & 0,5 \\
\hline
\end{tabular}

Fuente: Personal de enfermería pabellón Fundadores A, junio - octubre 2004. 
disponer y aplicar el conocimiento como elemento para vencer las barreras que se puedan presentar, con el fin de alcanzar objetivos en el aprendizaje. A través del ejercicio teórico se aprende y se adquiere una aptitud por la práctica, lo cual modifica la conducta anterior, afirmando utilidad para sí mismo y la comunidad. La desviación estándar con un puntaje de 0,5 refleja que hay aspectos que deben ser reforzados en el grupo participante como la inclusión de la familia y la verificación del proceso educativo al paciente.

\section{Conclusiones}

El diseño y aplicación del modelo educativo mejoró la práctica educativa del personal de enfermería, hacia el paciente quirúrgico sobre los cuidados en casa con la herida, lo que demuestra una base adecuada para continuar con los refuerzos hasta obtener su estandarización. La práctica educativa del personal de enfermería es susceptible de estandarizarse a nivel institucional para todo tipo de pacientes, con el despliegue del modelo descrito en el estudio.

\section{Agradecimientos:}

Agradecemos a la enfermera Patricia Arroyo, Jefe del Departamento de enfermería del HSJ, por la credibilidad que otorgó a la investigación como parte del proceso de calidad que encamina esta institución; al personal de enfermería del pabellón Fundadores A por su tiempo y dedicación al modificar el proceso educativo permitiendo cumplir los objetivos del mismo, a la docente Janneth Peñuela por hacer que este proyecto cumpliera con las normas establecidas en la presentación escrita. Por último al Hospital de San José por el apoyo logístico y por brindar al grupo investigador un campo de aplicación para la búsqueda de modelos que permitan trabajar unidos hacia la excelencia del cuidado de nuestros pacientes.

\section{Lecturas recomendadas}

Uniovi definición de términos. 2002. (25 de ag.2004) Disponible en: http://tradu.scig.uniovi.es/trad.html,

Moreno R. Calidad de educación recibida por los pacientes en postoperatorio sobre los cuidados en casa con herida quirúrgica en una IPS de tercer nivel. Bogotá: Fundación Universitaria de Ciencias de la Salud. Facultad de Enfermería, 2003. $18 \mathrm{p}$.

Florez Hurtado PA et al. Conocimientos, actitudes y prácticas del personal de enfermería sobre los cuidados en casa con la herida quirúrgica en el Hospital San José, Tesis. Bogotá: FUCS. Fac. de Enfermería 2004.

Hernández Sampieri R. Metodología de la Investigación. 3 ed. Madrid: Mc Graw Hill, 2003 p 368-84

Papalia E. D, Rally Wendkos O. Psicología del desarrollo: Skinner condicionamiento operante. 7 ed. México DF: Mc Graw Hill, 1993. 32p.

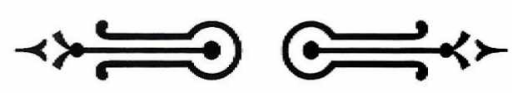

